\title{
PROGRAMA INTERDISCIPLINAR QUE ATIENDE A LA EDUCACIÓN PARA EL OCIO, EL CONSUMO Y EL MEDIO AMBIENTE BASADO EN LA PRODUCCIÓN DE MATERIALES DIDÁCTICOS Y LÚDICOS DE EDUCACIÓN FÍSICA
}

\author{
Ana Ponce de León Elizondo \\ Esther Gargallo Ibort \\ $M^{\mathrm{a}}$ Carmen de Lemus Varela \\ Ma Pilar Treviño Fernández \\ Edmundo Loza Olave \\ $M^{\mathrm{a}}$ Teresa Pascual Sufrate \\ Ma Luisa Fernández Armesto \\ Universidad de La Rioja
}

\begin{abstract}
RESUMEN. Exponemos el contenido de un programa educativo que evaluamos empleando estrategias de investigación-acción.

Constituye un recurso contrastado que puede ser empleado tanto en educación formal como en educación no formal y que pretende potenciar las actitudes positivas hacia el medio ambiente, el consumo responsable, la cooperación o el empleo constructivo del tiempo libre. Todo ello desde una perspectiva interdisciplinar que gira en torno a la construcción de recursos materiales de educación física a partir de materiales de desecho.
\end{abstract}

ABSTRACT. We explain the contents of an educative programme that we evaluate with some strategies of action-research.

This is a checked resource that can be used in Formal Education as well as in informal one. It seeks to develop positive attitudes towards the environment, the responsible consumer, the cooperation or the positive occupation of free time. All this is explained from an interdisciplinary perspective that turns round the material resources= building of physical education starting from waste products.

\section{Introducción}

Este proyecto interdisciplinar surge de la inquietud y coincidencia de criterios ante la demanda que se hace por parte de los maestros. Partimos de una inquietud de búsqueda y compromiso con la calidad educativa de los profesores que forman el equipo investigador. Esa inquietud y ese compromiso se hacen realidad en este programa, 
en el que ofrecemos un material que pueda facilitar la tarea educadora si ésta pretende educar las actitudes y potenciar las experiencias globalizadas.

El programa puede ser aplicado por los maestros en el centro escolar, con el fin de que sea la escuela quien ofrezca recursos para la utilización constructiva del tiempo libre y la que favorezca actitudes positivas hacia el medio ambiente, el consumo responsable y la creatividad.

Coincidimos con Gutiérrez Pérez (1995) en un concepto de la transversalidad contemplada no sólo como eje fundamental para objetivos y contenidos sino como otra vía de comunicación que la escuela puede establecer con el exterior para ofrecer soluciones educativas compartidas con otras instituciones del entorno cuya función educadora cada vez adquiere más importancia (ludotecas, colonias de vacaciones, atención a minorías, etc. ).

La meta a conseguir ha sido diseñar y evaluar un programa que implique diversas áreas del currículo básico, así como las transversales. Partimos de ideas que diferentes autores como Tonucci, F.(1988), González Faraco, J.C. (1997), Arca,J., Delpuy, E., Llanas, T. y Roy, Y. (1997) han tratado relacionando el juego con la Educación Ambiental, la creatividad, la cooperación y la igualdad de oportunidades.

Es preciso dar una respuesta ágil a las demandas de una sociedad con problemas dando paso a una modalidad de aprendizaje menos directivo y académico que se apoya en cualquier tipo de metodología desde las tecnologías más avanzadas hasta los materiales más rudimentarios (Gutiérrez Pérez, 1995).

En la propuesta que hacemos se ofrece a los alumnos la posibilidad de fabricar juguetes empleando materiales de desecho.

El uso de materiales de desecho favorece la concienciación hacia la recogida selectiva. La búsqueda de los envases adecuados, la apreciación de sus dimensiones y composición así como de la frecuencia con que se tiran y el volumen que representan incide claramente en estas actitudes.

La reutilización de papel y plástico, 32 \% del cubo de basura en España según el MOPMA (1996), en estas actividades mentaliza ante la evidencia del alto volumen que suponen los residuos urbanos. La gestión integral de los residuos es, hoy por hoy, una filosofía. Llegará a ser realidad sólo a través de soluciones locales que dependen de la cultura, la aceptación por parte de la población, la disponibilidad de mercados para materiales reciclados, las infraestructuras, el clima y la densidad de población (Barrenechea, P.; Larruga, S. y Varea, M., 1997).

Consideramos que la reutilización de envases y otros materiales desechables da a los niños y niñas la posibilidad de contribuir a la tarea de minimización de residuos pero también de reconocer el uso humano de un objeto que ha requerido en muchos casos un alto costo en recursos materiales y energéticos.

Cuando probamos las posibilidades de cortar, pintar, encajar o medir que tienen por ejemplo, los botes de yogur o las cajas de cartón de diferentes tamaños, estamos apoyando la idea planteada por F. Tonucci: "Pero la caja tiene una historia propia, era una caja de algo, contenía algo y ya ha terminado su función, ya no sirve y se ha desechado; para utilizarla de nuevo es preciso saber olvidar esta historia, descubrir lo 
nuevo en lo viejo, saber ver la caja con una nueva mirada, como un objeto disponible para realizar funciones nuevas, nuevos significados."

La creatividad, el trabajo en equipo y las habilidades manuales y técnicas tienen un fructífero campo de aplicación cuando los grupos están atareados en el empeño de ensamblar adecuadamente las piezas de ese material que esperan con ilusión que se transforme en un juego que resulte bonito y eficaz, y un vulgar envase, con pinturas, unas hebras de lana y unos trozos de cinta aislante se convierten en una pequeña pieza de arte. Luego, al ser probadas, aparecen distintas dificultades técnicas que les hacen pensar de nuevo cómo introducir modificaciones que los hagan más duraderos y eficaces a la hora de jugar o de hacer ejercicios físicos.

\section{Antecedentes}

Este trabajo recoge las aportaciones de diversos colectivos relacionados con la Educación: alumnos, profesores, padres y alumnos de Magisterio. Los autores pertenecemos a cuatro áreas de conocimiento diferentes, pero todos impartimos docencia en la diplomatura de Maestro en las especialidades de Educación Infantil, Musical, Lengua Extranjera y Educación Física. Hemos recibido el apoyo de la Universidad de La Rioja con una ayuda para el desarrollo de un proyecto de investigación durante dos años y del Instituto de Estudios Riojanos con un proyecto anual.

Con el fin de conocer las posibilidades y las necesidades de los centros educativos, respecto del tema que teníamos planteado, se elaboraron y analizaron unas encuestas donde se solicitaba a una muestra de profesores y profesoras de Primaria y Secundaria de Centros públicos y privados, rurales y urbanos, su opinión sobre cinco bloques de ítems:

- Integración del tiempo libre en el proceso educativo.

- Creatividad, objetivos y técnicas.

- Implicación de las áreas transversales en la programación y desarrollo educativo.

- Actitudes de los alumnos y alumnas hacia las áreas transversales.

- Utilización de diferentes juegos y materiales en Educación Física.

Veinte alumnos y alumnas de Magisterio de la especialidad de Educación Física han compartido con el equipo un Seminario durante el curso 97-98 preparando una serie de materiales así como las posibilidades de juego que estos pueden ofrecer. (EI desarrollo y conclusiones del mismo se ofrecen en Ponce de León A., Gargallo E., Lemus, M.C., Loza E., Fernández M.L., Treviño M. P. y Pascual M.T., 1999 b)

Como resultado de esta parte de la experiencia seleccionamos 32 materiales fijando las pautas para su construcción, uso y tipos de juegos que pueden llevarse a cabo con ellos. Para su validación en los niveles básicos de enseñanza, se experimentaron esos materiales con 73 alumnos y alumnas de $6^{\circ}$ de Primaria de un centro urbano de Logroño y otro rural con resultados muy favorables contando con la colaboración activa de profesores y profesoras encargados de curso o de área. La síntesis de esta etapa se recoge en el libro Reciclo, construyo, juego y me divierto, Una propuesta interdisciplinar para la Educación del Ocio, el Consumo, el Medio Ambiente y la Educación Física (Ponce de León, A y Gargallo, E -coord.-, 1999). 


\section{Objetivos}

1. Mejorar o afianzar la confianza y la relación entre alumno y profesor al trabajar como iguales en la elaboración del material y participando en el juego.

2. Dar posibilidades a los niños y jóvenes para que descubran, a través de la construcción de sus propios juegos y materiales lúdicos, alternativas en su tiempo de ocio que potencien la creatividad, la cooperación y la actividad física.

3. Favorecer un clima que cuestione la enorme red de consumo que rodea al mundo del ocio y muestre una posible vía que permita a cada uno ocupar su tiempo libre con alternativas no consumistas.

4. Responsabilizarse ante el grave problema del volumen que suponen los residuos y la oportunidad de conocer formas de reutilización, reciclaje y reducción.

5. Plantearse interrogantes sobre la necesidad de los envases para mejorar la calidad de vida.

6. Sensibilizar a los padres en actuaciones favorables hacia el Medio Ambiente y el consumo responsable a través de la implicación en las actividades de sus hijos e hijas.

7. Abrir cauces hacia el fomento de la innovación educativa en los profesionales de la enseñanza de centros no universitarios que asuman el trabajo coordinado de diferentes especialistas y valoren la importancia de que la Comunidad Educativa desarrolle proyectos de innovación que, iniciándose en el contexto escolar, tengan una continuidad en el medio familiar y social.

\section{Desarrollo del programa}

El programa está basado en la construcción de juegos elaborados a partir de materiales de desecho acompañados de accesorios de bajo costo y elementos decorativos. Igualmente se elaboran las correspondientes actividades regladas y normativizadas para el uso de los juegos de forma individual y en grupo.

Se desarrolla en cinco sesiones y se ha experimentado en $6^{\circ}$ curso de Educación Primaria.

Para el seguimiento de la experiencia se han preparado, aplicado y evaluado una serie de cuestionarios a los padres, a los profesores colaboradores en las aulas y una autoevaluación final de los alumnos y alumnas que han participado en el programa. Los cuestionarios y las pautas para la observación figuran como anexos.

No se ha pretendido una gran perfección de los juegos conseguidos; el acento se ha puesto en el proceso, en la resolución de problemas manipulativos, en las relaciones interpersonales que surgen entre los integrantes del grupo, en el empleo de instrumentos de medida, en la asunción de tareas y en la participación en la toma de decisiones.

Las sesiones tienen una duración de una hora y media o dos horas y se llevan a cabo unas en el aula y otras en el gimnasio, y quedan distribuidas como sigue:

1a sesión: ¡No lo tires, puede ser útil!

2a sesión: Experimentando nuevos materiales. 
3a sesión: ¿Cómo es nuestro proyecto de trabajo?

4- sesión: Construimos nuestros juguetes

5-a sesión: Jugamos y establecemos normas de juego

Paralelamente al desarrollo del programa se pueden desarrollar multitud de actividades, en diferentes áreas de conocimiento, que pueden tener relación con este centro de interés como:

- Diseñar campañas publicitarias

- Elaborar presupuestos, listas de pedidos y facturas. (Posibilidades de descuento....)

- Traducir a otros idiomas los proyectos, etc.

\section{SESIÓN № 1 \\ ¡NO LO TIRES, PUEDE SER ÚTIL!}

Lugar: Aula Duración $1 \mathrm{~h} 30 \mathrm{~min}$

Observadores: 2 investigadores, dos alumnos de prácticas y 1 profesor del centro

Objetivo de la sesión:

- Analizar los efectos sobre el medio ambiente de las acciones humanas, en concreto la acumulación de residuos sólidos urbanos.

- Valorar la importancia que tienen las actuaciones individuales responsables para la conservación del medio ambiente.

Áreas implicadas:

- Conocimiento del Medio

- Educación Ambiental

- Educación para el consumo

- Educación para la convivencia

Material:

- Bolsa con basura

- Productos con embalajes diversos

- Diferentes envases

Desarrollo:

Agrupamiento: Gran grupo

- Se inicia la sesión con una actividad-provocación: se abre una bolsa de basura y se analiza su contenido. Como suscitará comentarios diversos, se derivará la conversación hacia una clasificación de los restos. Los criterios pueden ser:

- Tipo de materiales: tela, papel, metal, pilas, vidrio, plástico, materia orgánica...

- Materiales reciclables/no reciclables. Materiales reutilizables / no reutilizables

- Se compara el volumen del material de la bolsa que puede ser reciclado en nuestra ciudad con el que va a parar al vertedero. 
- Se insiste en el tratamiento de ciertos residuos potencialmente peligrosos

- Se plantean destinos solidarios para objetos usados (ropa, gafas, radiografías....).

- Obtención de conclusiones:

- La acumulación de residuos es un problema grave.

- Debería haber algo que nosotros pudiéramos hacer.

- Se informa a los alumnos de las posibilidades que tenemos los ciudadanos para colaborar en la reducción de basuras:

RECICLAR: Depositar los residuos en su contenedor correspondiente.

REDUCIR: Comprar productos que tengan poco embalaje o que sea reciclable.

REUTILIZAR: Dar utilidad a objetos que normalmente tiramos a la basura.

- Se analizan las posibilidades de RECICLAJE que tenemos en nuestra ciudad haciendo una lista de los tipos de contenedores que podemos encontrar en nuestras calles y los materiales que podemos depositar en ellos.

Agrupamiento: Pequeño grupo

- Se proporciona a cada grupo una serie de productos habituales en los supermercados, para que analicen el tipo de envase, la información que da, las ventajas que aporta a la conservación del producto . Con esos datos deben decidir cuáles comprarían y cuáles no teniendo en cuenta que deben REDUCIR la cantidad de basura no reciclable.

- Se proporciona a cada grupo una serie de envases para que piensen qué uso alternativo podría tener cada uno de ellos. Se trata de que puedan ser REUTILIZADOS, alargando con ello su vida útil.

Agrupamiento: Gran grupo

- Se comentarán las ventajas que podríamos encontrar en la reutilización y reciclado de materiales y de objetos:

- Contribución a la mejora concreta en cuanto a la estética y a la higiene del medio.

- Ahorro de materias primas y energía en la fabricación de envases.

- Desarrollo de la creatividad.

- Empleo del tiempo de ocio de manera constructiva.

- Hábitos para un consumo más responsable.

- Como final de la sesión se les daría información sobre el trabajo que van a realizar. 


\section{SESIÓN № 2 \\ EXPERIMENTANDO NUEVOS MATERIALES}

Lugar: Gimnasio Duración: 1 h 30 min.

Observadores: 2 investigadores, 2 alumnos de prácticas y 1 profesor del centro

Objetivo de la sesión:

- Experimentar nuevos materiales de Educación Física

- Analizar su construcción

Áreas implicadas:

- Educación Física

- Conocimiento del Medio Natural

- Educación para la convivencia

Material:

- 6 juguetes elaborados en el seminario. A ser posible se elegirán aquellos que exijan un mayor componente motor.

Desarrollo:

Suponemos que en cada una de las clases hay un número aproximado de 24 alumnos. La clase se dividirá en 6 grupos de 4 alumnos cada uno.

El material se distribuirá en 6 bases o postas con juguetes diferentes. En cada una de ellas habrá un juguete para cada alumno (con todos sus juguetes iguales).

Los alumnos permanecerán 15 minutos en cada una de las bases experimentando libremente con el material.

En los 5 primeros minutos se les explicará a los alumnos la organización de la clase y la forma ordenada de circular entre las bases (a la señal propuesta cada grupo se desplazará en el sentido de las agujas del reloj).

En cada una de las bases habrá un observador. Se registrarán las variantes establecidas para la observación. La forma de recoger la información se basará en el diálogo con cada uno de los grupos que pasen por las bases (el investigador puede ir realizando preguntas a cada alumno mientras éstos experimentan con el material). 


\section{SESIÓN № 3 \\ CÓMO ES NUESTRO PROYECTO DE TRABAJO}

Lugar: Aula Duración: 1 hora

Observadores 1 investigador, 2 alumnos de prácticas y 1 profesor del centro

Objetivo de la sesión:

- Elaborar un documento en el que se especifiquen las características del objeto a construir, los materiales y las herramientas necesarias y los pasos que se han de dar en la construcción.

Áreas implicadas:

- Lenguaje

- Educación Plástica

- Educación Física

- Educación para la Convivencia

- Conocimiento del Medio

Desarrollo:

Deben diseñar un juguete que pueda ser utilizado en las clases de Educación Física empleando para ello objetos (envases o materiales) que se reutilizan en lugar de tirarlos a la basura.

Las actividades que los alumnos puedan hacer con ellos tendrán que ver con los temas que se trabajan habitualmente en Educación Física: carreras, saltos, equilibrio, lanzamientos, etc.

El trabajo de cada grupo (pequeño grupo) seguirá estas fases:

- Diseño del proyecto. Deben determinar en el grupo qué aspectos deben aparecer en ese documento, de modo que si otro grupo debiera hacerse cargo de su construcción, el resultado fuera el mismo que si los construyeran ellos. Tras una puesta en común, el esquema de trabajo sería éste:

- Descripción del juguete

- Dibujos

- Materiales y herramientas a emplear

- Proceso de construcción

- Puesta en común de las propuestas de juguete de cada miembro. Pueden añadir dibujos a sus explicaciones.

- Toma de decisiones teniendo en cuenta diversos factores como el nivel de dificultad, el acceso a los materiales y las herramientas, sus posibilidades de uso en sesiones de Educación Física, etc. Deberán acordar la construcción de uno de los juguetes propuestos.

- Dibujo del juguete, se presentarán los planos más adecuados ( perfil, planta....)

- Descripción del juguete. Explicación de sus posibles usos.

- Especificación de las herramientas y los materiales necesarios.

- Desarrollo de las fases de construcción. Reparto de tareas en cada una de ellas.

Todos estos apartados constituirán el documento del "Proyecto", que dirigirá la fase posterior de construcción. 


\section{SESION № 4 \\ REALIZANDO NUESTROS PROPIOS MATERIALES}

Lugar: Taller de Plástica.

Duración: 2 horas

Observadores:2 investigadores, 2 alumnos de prácticas, 1 profesor del centro.

Objetivo de la sesión:

- Realizar nuevos materiales de Educación Física a partir de otros reutilizables, siguiendo el proyecto elaborado anteriormente.

Áreas implicadas:

- Educación Plástica - Matemáticas

- Educación Física - Lenguaje

- Conocimiento del Medio - Educación para la Convivencia

Material:

- Material reutilizable aportado por los alumnos.

- Materiales básicos y herramientas: tijeras, cinta aislante, pegamento de contacto, pinturas, pinceles, recipientes para mezclas, etc. (estarán en función del juguete que vaya a elaborarse)

Desarrollo:

- Se dan a los alumnos una serie de pautas sobre el manejo de herramientas, así como pautas sobre orden y limpieza.

- Posteriormente los alumnos organizados en los grupos que se crearon en la sesión anterior comienzan la construcción de su juguete siguiendo las especificaciones que plasmaron en el proyecto.

- Elaboración de una memoria en la que se reflejen los pasos que se han dado para la construcción del juguete, los materiales que se han utilizado tanto reciclables como básicos, los utensilios, el tiempo que les ha llevado en la elaboración, las variantes y aplicaciones a nuevos juegos.

Breve experimentación del material y revisión si fuese necesario.

\section{SESION № 5 \\ JUGAMOS Y ESTABLECEMOS REGLAS DE JUEGO}

Para llevar a cabo esta sesión destinada a que los alumnos jueguen con los artefactos que han construido, se deja libertad al maestro para que pueda organizarla en función de cada circunstancia. Las opciones que se ofrecen, sin pretender agotar las posibilidades son éstas:

- Llevar a cabo una sesión similar a la no 2 .

- Organizar competiciones con los juguetes.

- Llevar a cabo los juegos en alguna fiesta del colegio... 


\section{Evaluación del programa - conclusiones}

Para la evaluación cuantitativa y cualitativa del programa se han utilizado distintos instrumentos como la observación y los registros anecdóticos, el diario de clase, grabaciones en vídeo, entrevistas y cuestionarios al profesor y cuestionarios a los padres previo y posterior a la aplicación del programa. Estos nos han permitido conocer no sólo el impacto o resultado de una intervención o comprobación de hipótesis sino también describir estos procesos, entender los resultados y explicar sus mecanismos causales. Al mismo tiempo, se obtiene información sobre cómo se está llevando a cabo el tratamiento de algunos temas transversales en los centros, el nivel de concienciación que en el entorno familiar se tiene actualmente con respecto a estos temas involucrados en el programa y la viabilidad de este tipo de experiencias en los centros educativos.

El programa ha incluido igualmente una autoevaluación a realizar por los alumnos participantes, con la cual se pretende abordar algunos aspectos de carácter actitudinal como el nivel de compromiso y actitudes en el trabajo individual y colectivo.

A la vista de las diferentes fases de la investigación y de los distintos ámbitos sobre los que incidía el programa podemos extraer las siguientes conclusiones.

A. Sobre el tratamiento educativo que los profesores dan a los temas implícitos en el programa, previo a la aplicación del mismo, -educación para el ocio desde la escuela, educación del medio ambiente, del consumo, interdisciplinariedad y creatividad.

Los profesores de Primaria y Secundaria encuestados (el 76,4\% de centros públicos de La Rioja y el 23,6\% de centros privados), tienen una media entre 16 y 17 años de servicio a la docencia.

Al preguntarles sobre si era competencia de la escuela el suscitar intereses en los alumnos para que ocupen su tiempo libre en algo que les forme y construya como personas, el $97 \%$ de los maestros consideran que es competencia de la escuela todo aquello que forme parte de la educación global que ayude a un mejor desarrollo del alumnado; por ello afirman, además, que la Educación para el tiempo libre tiene un lugar en la institución escolar por las siguientes razones:

- Es un aspecto más de la vida que puede y debe ser educado, al mismo tiempo que es una exigencia de la sociedad para el desarrollo de la persona al constituirse ese tiempo de ocio, gratificante y creativo, en vehículo de contacto entre gentes y culturas.

- El profesorado de Educación Física piensa que debe contribuir a crear actitudes positivas hacia el deporte y la actividad física fomentando hábitos saludables que aparten a los alumnos de ambientes insanos (droga, alcohol,...) y, en definitiva, mostrar las ventajas de la utilización del tiempo libre de manera adecuada.

- Para muchos profesores, la educación para el tiempo libre debe partir tanto de las áreas del currículo como de los temas transversales, valorando las actividades que realizan, creando inquietudes y fomentando actitudes positivas.

Sin embargo, también resaltan que la educación para el tiempo libre no es exclusiva de la escuela, la familia y otras instituciones deben estar implicados. 
Se quiso conocer igualmente si los diferentes Claustros se involucraban en proyectos en relación con el tiempo libre donde se implique conjuntamente a todo el profesorado. La mayor parte de los profesores reconoció que la escuela debería dar importancia a la educación para el tiempo libre pero, muy a pesar de ello, la realidad es bien distinta y basada tan sólo en programar determinadas salidas y actividades culturales que no dejan de ser cuestiones puntuales y aisladas y no como un trabajo y proyecto conjunto, a pesar de que en ellas se impliquen a padres, profesores y alumnos y estén incluidas en el PEC.

Sobre la forma de integrar la educación para el tiempo libre en los contenidos de las diferentes áreas, los profesores destacan que debería tratarse desde las diferentes áreas pero desconocen la forma de llevarlo a la práctica.

Otro de los objetivos a los que responde nuestro programa es la creatividad, en la encuesta se les preguntaba si en el PEC se contempla la creatividad como un objetivo a desarrollar en todas las áreas, ellos señalan que es un objetivo que se queda sobre el papel o se trabaja únicamente desde el área de Educación Plástica.

Desde el ámbito de la Educación Física los alumnos buscan las posibilidades de utilización de los materiales empleados en clase o sugieren nuevos juegos y variantes de las actividades ofertadas por el profesor. Los contenidos relacionados con la expresión también suponen un medio para trabajar la creatividad. Al mismo tiempo, los profesores de algunas áreas utilizan proyectos para que los alumnos creen sus propias actividades.

Por otra parte, de las encuestas se deduce que los profesores nunca han construido materiales de Educación Física, que son muy pocos los que se implican de manera concreta en actividades de reutilización, ya sea por falta de tiempo, por necesidad de asesoramiento técnico, por ausencia de recursos didácticos o por desconocer la forma concreta de llevarlo a cabo.

Más de la mitad del profesorado (60\%) reconoce que es "mínimo" el grado de implicación que tiene el tema transversal de la Educación Ambiental en la programación y en el desarrollo educativo de su área y que éste se concreta puntualmente y en primer lugar, en salidas, denominadas "actividades al aire libre", las campañas de reciclado en segundo, las semanas del árbol, cuidado, limpieza y conservación del Colegio en tercero y, por último, las charlas, talleres de papel y uso de material audiovisual específico en las que se llega a implicar también a la familia.

La identificación de la Educación Ambiental con las salidas a "conocer la naturaleza" está muy marcada mientras que actividades más próximas, como reciclado, limpieza y conservación del Colegio, que se pueden hacer siempre, son menos reconocidas. Concluyendo, se considera que el mayor grado de implicación se da en el área de Conocimiento del Medio, pero aunque pueda ser la más adecuada, en cuanto a contenidos conceptuales, no debe ser la única.

Teniendo en cuenta los datos reflejados en el gráfico, parece ser que el grado de implicación del tema del Medio Ambiente mejoraría con asesoramiento y materiales adecuados y una flexibilidad de horario para que los profesores pudieran preparar y realizar las actividades. 


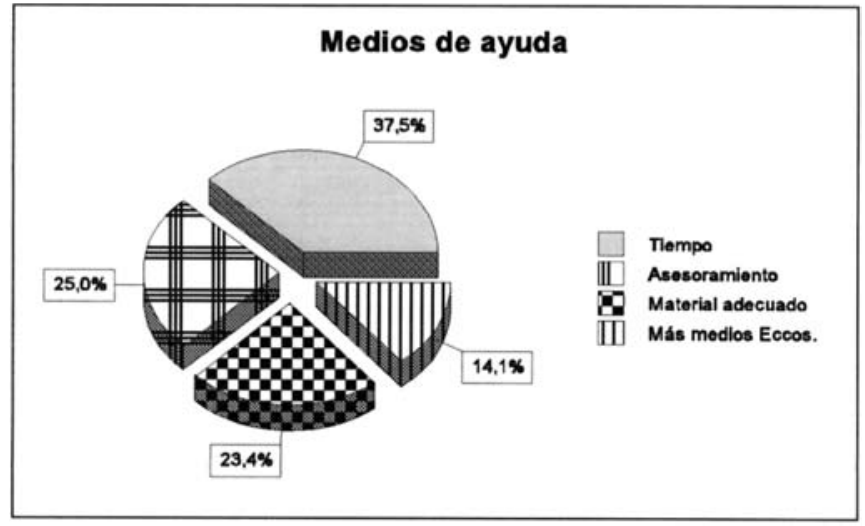

En el gráfico siguiente se observa que un significativo porcentaje de profesores trabajan con material de desecho en sus clases, concretamente el 75,5\%, frente a un $22,2 \%$ que no los utiliza.

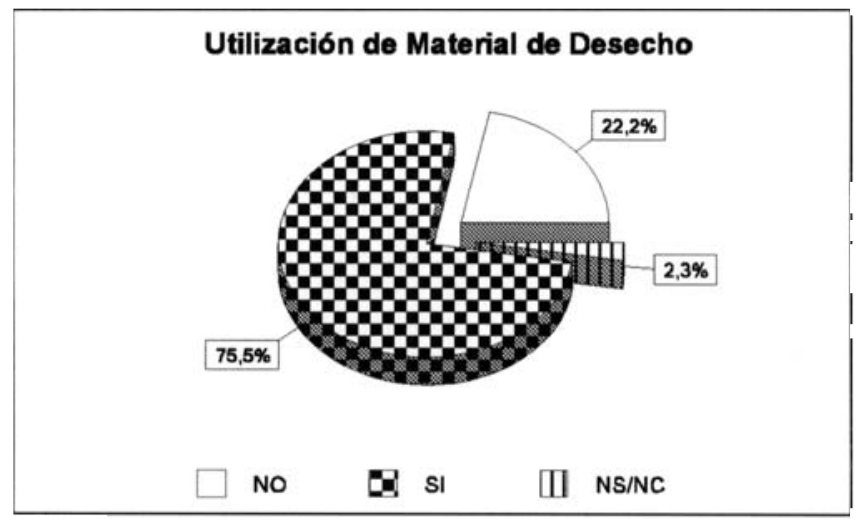

La lista de los materiales de desecho que emplean es bastante amplia, destacan como los más citados: cajas, botellas y botes de plástico, papel, periódicos, latas, lanas y chapas. Además se usan: botones, postales, fotos, tubos de cartón, neumáticos de coche o bici, aros, hueveras, latas de Coca-Cola, madera y cuerdas.

El uso que se da es para construir: instrumentos musicales, disfraces, trabajos manuales y juegos. También para hacer ejercicios de coordinación, saltos, lanzamientos y fabricar papel reciclado.

Además el 87,5 \% de los profesores, como se constata en el gráfico, considera que la utilización de materiales de desecho y su reutilización en el aula favorece el desarrollo de una actitud ciudadana frente a un insignificante $3,1 \%$ que no lo estiman así y un $9,4 \%$ que no contestan. 


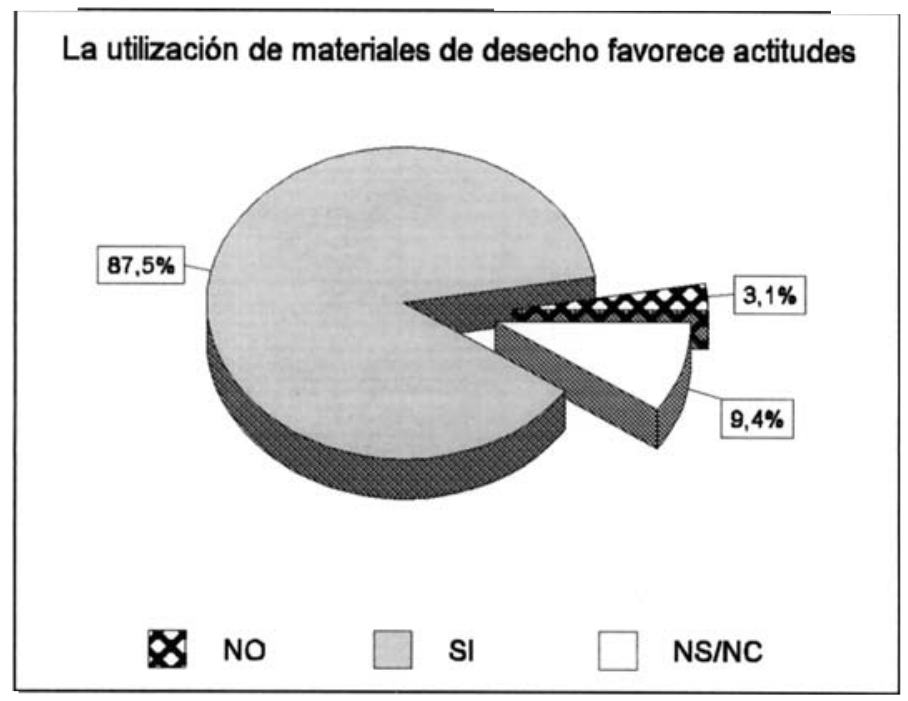

B. Sobre las actitudes familiares en cuanto a la recogida, selección y utilización de envases u otros materiales de desecho.

Con el fin de aproximarnos a la realidad de las familias de los alumnos y alumnas que han tomado parte en este programa educativo solicitamos, previo a la aplicación del programa, la colaboración de los padres para conocer las actitudes hacia la minimización de residuos y el aprovechamiento de algunos de ellos en el seno familiar.

Los resultados constatan que el $76,6 \%$ tiene costumbre de clasificar los materiales de desecho, fundamentalmente papel, pilas, vidrio separadamente del resto, frente a un $13,4 \%$ que no acostumbran a separar los residuos.

El gráfico siguiente muestra la frecuencia con que se acostumbra a depositar los materiales de desecho, clasificados en casa, en los respectivos contenedores.

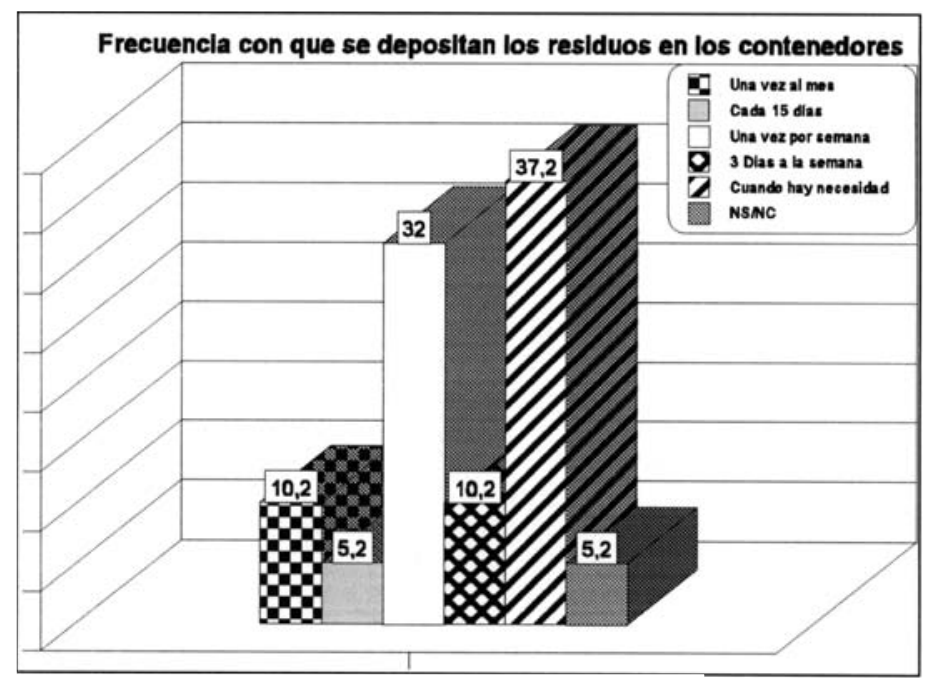



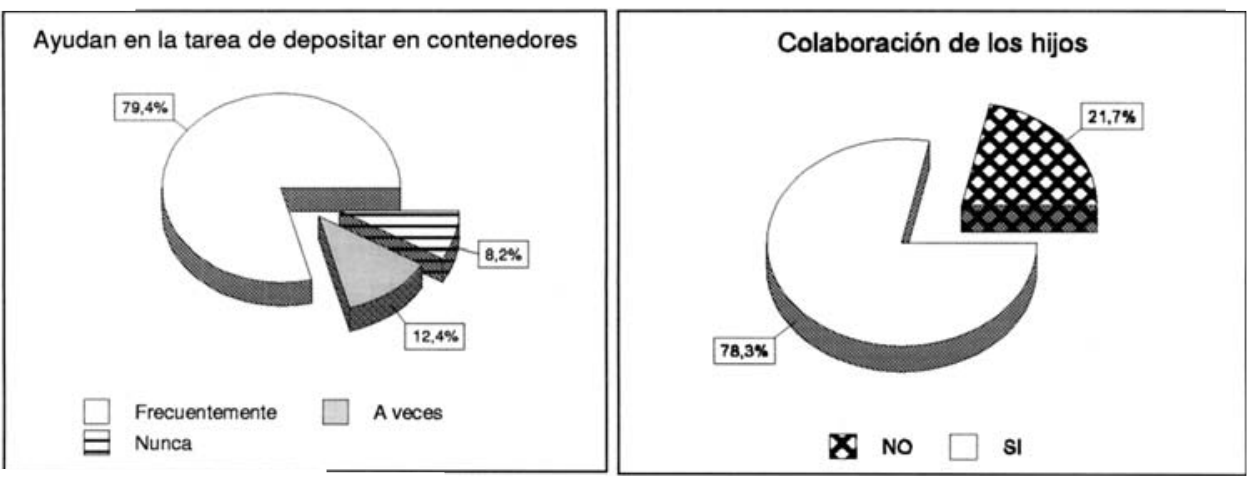

Como puede observarse en las gráficas en el 78,3\% de las familias, los hijos colaboran en las tareas de separar los residuos, mientras que en un 21,7\% no lo hacen. Así mismo, un 79,4\% acostumbra "frecuentemente" a mandar a los hijos a depositar los diferentes residuos en el contenedor correspondiente, un 12,4\% lo hace "a veces" y un $8,2 \%$ "nunca".
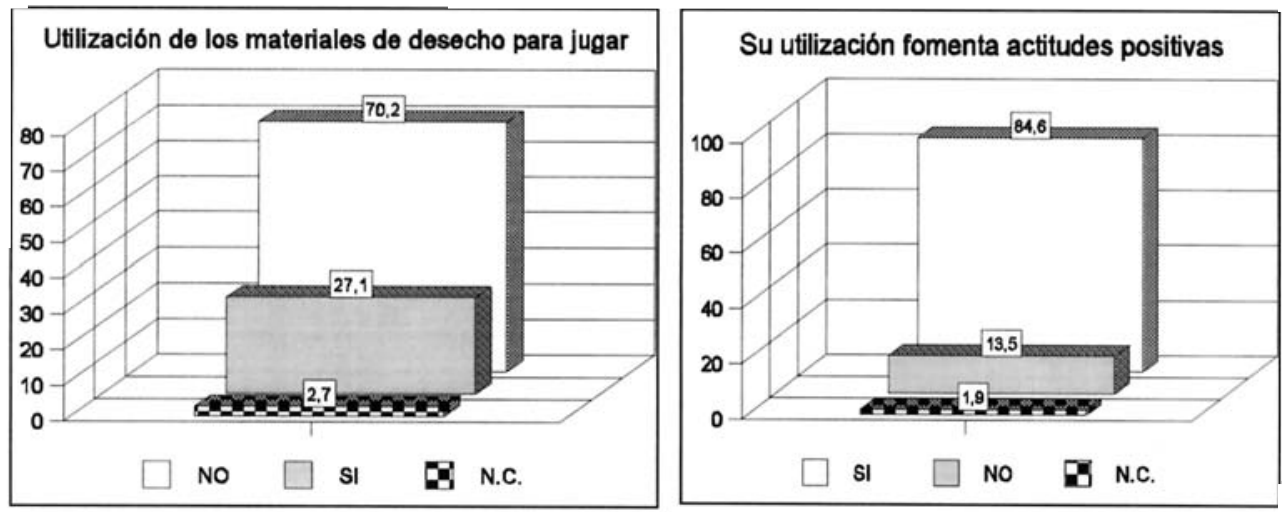

Un $81 \%$ de los padres manifiestan que sus hijos han jugado alguna vez con materiales de desecho aunque lo hacían más de pequeños. La proporción se invierte al comprobarse en $6^{\circ}$ de Primaria; frente a un $27,1 \%$ que siguen utilizando materiales de desecho, se encuentra un 70,2 \% que no utiliza estos materiales para jugar y un $2,7 \%$ no constesta.

A pesar de ello, un $84,6 \%$ de los padres creen que el trabajo con materiales de desecho y el juego utilizando estos tipos de materiales, pueden fomentar actitudes responsables hacia el medio ambiente, frente a un 13,5\% que dicen que no servirá y $1,9 \%$ que no contestan.

A las propuestas planteadas en la línea de nuestro programa, la gráfica confirma las inquietudes de los padres por la sensibilización hacia el Medio Ambiente y la reutilización de materiales con fines lúdicos; se constata que a un 78,3\% de los padres les gustaría poder participar en un taller de reutilización de residuos en la construcción de materiales junto a sus hijos, al $13,5 \%$ no le interesa y un $8,2 \%$ no contesta. 


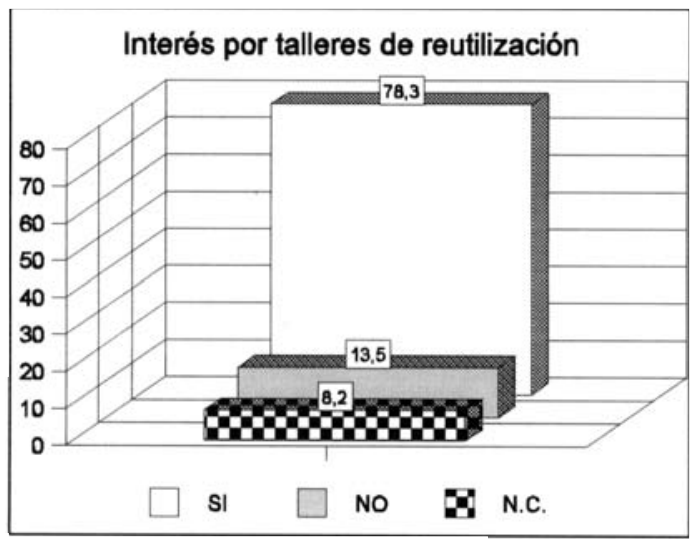

\section{C.- Sobre la aplicación global del programa y el desarrollo de las diferentes sesiones}

Si bien cada contexto escolar fue diferente, podemos extraer conclusiones generales en cuanto a la aplicación del programa y la consecución de los objetivos propuestos en el mismo.

El trabajo en equipo en los diferentes contextos proporcionó la posibilidad de cooperar para buscar soluciones conjuntas a problemas técnicos que requerían probar y desechar alternativas hasta encontrar la forma más eficaz de diseñar el material. Este hecho, como puede imaginarse, desarrolla en el alumno habilidades sociales y valores relacionados con el respeto, la tolerancia, la aceptación de diferentes puntos de vista, la capacidad de discusión en grupos, la asunción de tareas acordes a las destrezas individuales o la tenacidad de no abandonar ante el fracaso.

Al mismo tiempo desarrolla la creatividad, la inteligencia práctica, la lectura comprensiva, las habilidades matemáticas y las destrezas manuales relacionadas con la manipulación de utensilios.

La utilización del material de desecho da a los niños idea sobre minimización de residuos, de puesta en circulación dentro del campo del uso humano de un objeto que ha requerido, en muchos casos, un alto costo en recursos materiales y energéticos y una oportunidad de valorar un objeto que les pasa desapercibido. Cuando los alumnos prueban las posibilidades de cortar, pintar, encajar, medir, que tienen por ejemplo, las botellas de leche o las hueveras, están comprendiendo un poco mejor el desarrollo industrial que permite obtener esos materiales tan variados y su enorme utilidad para envasar alimentos u otros tipos de bienes de consumo.

En la situación de trabajo, cuando los grupos están enfrascados en conseguir ensamblar adecuadamente las piezas de ese juego que esperan con ilusión que resulte bonito y eficaz, un envase, unas hebras de lana o un trozo de cinta aislante, se convierten en enormes tesoros que, convenientemente disfrazados, pasan a ser pequeñas piezas de arte. Luego, al ser probadas, aparecen distintos defectos técnicos que les hacen pensar de nuevo en cómo introducir modificaciones para que sean más duraderos y eficaces a la hora de jugar o de hacer ejercicios físicos.

El hecho de elegir, para adornar las superficies, símbolos relacionados con los tipos de residuos y sus posibilidades de recogida selectiva, que en principio no parecía ser 
más que una cosa anecdótica, se reveló como otro acierto. Los niños tuvieron que esforzarse en recordar los tipos de contenedores, usar los términos correctos cuando dan órdenes a la hora de jugar en el tapiz o en el tragachapas con lo cual el aspecto medioambiental tiene un nuevo refuerzo.

La experiencia ha resultado positiva tanto por la implicación y coordinación de diferentes profesores y áreas como por la necesidad de búsqueda de materiales y la ejecución de "su juego".

La primera sesión, celebrada en el aula y denominada ¡No lo tires, puede ser útil!), ha perseguido fundamentalmente dar a conocer a los alumnos participantes el volumen de residuos sólidos urbanos que se generan en nuestros domicilios y la procedencia y naturaleza de los mismos.

Para ello se mostró una bolsa de basura en la que se encontraban distintos residuos proponiéndose la clasificación de los mismos. Se plantearon también las posibilidades de reutilización, reciclado y reducción de volumen, la importancia de los envases en la sociedad actual y la necesidad de un correcto uso de los mismos. Igualmente se señalaron los residuos peligrosos como medicinas, insecticidas que tienen un tratamiento especial y aquellos objetos que desechamos y pueden ser aprovechados por personas desfavorecidas, concenciándonos para entregarlos en organizaciones solidarias.

De entrada la mayor parte de los niños, a pesar de la curiosidad inicial que la novedosa propuesta de clase despierta, muestran ciertas reticencias a tocar y coger los materiales que la profesora extrae de las bolsas de basura, por lo que se hace necesaria la intervención del maestro para que comiencen a tomar contacto con los distintos materiales y los clasifiquen.

Resultó interesante para ellos considerar la trascendencia que tienen los comportamientos individuales respecto a la minimización de residuos, especialmente en el momento de la compra.

La segunda sesión, denominada Experimentando nuevos materiales, tuvo lugar en el gimnasio. Se pretendió, tal y como su nombre indica, experimentar y utilizar los nuevos materiales de Educación Física (concretamente alguno de los construidos en la primera fase de esta investigación).

A tal efecto, se distribuyó a la totalidad de los alumnos en varios grupos y, mediante un sistema de trabajo en circuito, todos fueron experimentando cada uno de los materiales mostrados en la sesión.

La primera tarea consistió en proponer nombres para cada uno de los materiales mostrados, la propuesta de algunos guardó relación con los materiales empleados en su construcción, pero mayoritariamente fue la utilidad del juguete la que determinó los distintos nombres que les asignaron ("encájala" al aro con botellas, "pasabola" al bote de suavizante). Incluso cuando con un mismo material cambian de actividad, modifican también el nombre del mismo como en el caso del aro con botellas al que inicialmente le denominaron "encájala", posteriormente pasó a ser "platillo volante", "bolos", etc.

A la hora de agruparse para observar los materiales, lo hicieron de múltiples maneras, pero generalmente comenzaron por hacerlo de forma individualizada para posteriormente hacerlo por parejas y finalmente en grupos más amplios. 
La riqueza y variedad de habilidades que se desarrollan a través de estas actividades quedan, a juzgar por lo apuntado por los distintos observadores, fuera de toda duda, recogiéndose entre las anotaciones de éstos las siguientes: desarrollo de la organización espacial, dominio corporal, coordinación, velocidad de reacción y, muy especialmente, los lanzamientos de precisión y las recepciones.

El interés mostrado por los niños participantes es alto, dada la atracción que los juguetes ejercen sobre ellos, observándose un tiempo de práctica casi total y escasos abandonos. Desaparece la pasividad que presentan ciertos alumnos ante la Educación Física favoreciendo la participación de todos.

Resultó frecuente observar cómo los niños cambiaban de juegos con un mismo material. A veces, seguido de propuestas de uso claramente desacertadas, que suscitaron alguna que otra discusión dado que no todos prefirieron el cambio de juego.

Los comentarios más significativos que realizaron durante la experimentación práctica, tuvieron que ver fundamentalmente con las dificultades e inconvenientes de uso que plantearon algunos de los materiales, casi siempre motivados por su tamaño en el caso de las pelotas, poco peso en el caso de las chapas, su fragilidad, etc.

En lo referente a la cuestión sobre qué otros materiales construirían a partir de los ya empleados, las respuestas en general han sido bastante escasas cuando no nulas. No obstante hemos recogido algunas como cabezas de muñecos y un planetarium con las pelotas y frisbis en el caso de los discos con botellas.

Finalmente en cuanto al material que mayor aceptación tuvo, podríamos decir que se observa una relación directa entre nivel de aceptación, la facilidad de uso y la comprensión de las reglas. Así, por ejemplo los "suaviboles", precisamente por ver rápidamente su aplicación (algo semejante a la cestapunta) y por resultarle original a los alumnos, fue uno de los juguetes que tuvo más éxito. Tras éste, aparecieron la indíaca por su fácil manejo, los discos y bolos, las pelotas malabares y la pelota loca. Por su parte el tragachapas, dado que exige cierto nivel de precisión, es señalado como el de menor aceptación.

En cualquier caso, la conclusión más relevante de esta segunda sesión, además del alto nivel de motivación mostrado en todo momento por los alumnos, es que éstos, tras haber efectuado diversas actividades con los distintos materiales mostrados, quedan plenamente convencidos de que, efectivamente, con unos productos que en principio estaban destinados a la basura, se pueden practicar multitud de juegos y que, por tanto, hay que replantearse seriamente su reutilización. Entendemos que de este modo quedan conseguidos algunos de los objetivos fijados en nuestra experiencia.

La tercera sesión, denominada Cómo es nuestro proyecto de trabajo, se realizó nuevamente en el aula, en ella los alumnos, por grupos, elaboraron un documento descriptivo o proyecto de juguete en el que aparece toda la información que creyeron necesaria: descripción, dibujos, proceso constructivo, herramientas y materiales necesarios.

Las capacidades que los alumnos pusieron en juego fueron mútiples, tanto en aspectos referidos a las habilidades sociales (había que discutir, argumentar, tomar decisiones, ser respetuoso....), como a las habilidades lingüísticas (el documento podría ser empleado por otro grupo para construir el juguete), como a la expresión plástica (dibujos explicativos), o a la capacidad de anticipar y planificar la acción. 
Todos los alumnos sin excepción se implicaron en el trabajo de grupo. Cada uno aportó al menos una idea para proponer al grupo, y todo el mundo participó en la toma de decisiones. Los alumnos afirman haber respetado en todo momento las opiniones de los demás.

Al enjuiciar el trabajo de grupo los alumnos detectan, aunque de forma muy minoritaria, algunas dificultades que pasamos a analizar:

- A la hora de elegir el juguete que iban a fabricar, en los casos en que había más de una propuesta, se han inclinado por una votación como sistema más objetivo y justo. En otros casos han resuelto el conflicto elaborando más de un juguete, o bien han consensuado su elección en función de la facilidad/dificultad para encontrar materiales de desecho o las posibilidades de juego del material que fabricarían.

- Algún grupo no ha organizado bien el trabajo debido la existencia de desavenencias entre los miembros. Así unas personas han trabajado más que otras, o han aportado más materiales.

La cuarta sesión, denominada Realizando nuestros propios materiales, se desarrolló en el taller de plástica del colegio; en ella los alumnos, en función del proyecto de juguete diseñado en la sesión anterior, construyeron por grupos lo proyectado.

Merece la pena señalar que la actividad a lo largo de esta sesión fue altamente constructiva, ya que hubieron de hacer frente a multitud de problemas y resolverlos poniendo en juego todas sus posibilidades. Sin ser exhaustivos, señalaríamos varias situaciones generadoras de conflictos cognitivos que han propiciado aprendizajes:

- Resolución de problemas de cálculo (ajuste de medidas, reparto de piezas....)

- Resolución de problemas geométricos ( tamaño del radio para que encaje....)

- Resolución de problemas tecnológicos (cómo sujetar dos piezas, con qué herramienta cortar, cuál es el material más adecuado.....)

- Resolución de conflictos en el seno del grupo en caso de desacuerdos.

El nivel de satisfacción respecto del trabajo personal ha sido elevado ya que, según afirman los alumnos, han participado de manera positiva a lo largo de todo el proceso.

En líneas generales la percepción que han tenido los alumnos de su trabajo en grupo ha sido gratificante. Los motivos que aducen son estos:

- Ha sido divertido

- Cada uno hacía su tarea

- Se han encontrado a gusto con el grupo

- Han colaborado y se han ayudado unos a otros

La gran mayoría de alumnos no proponen cambio alguno para esta actividad. Los que lo hacen se refieren fundamentalmente al uso de otros materiales o a cambios en el juguete a fabricar en el sentido de elaborar algo más complicado.

El nivel de motivación ha sido elevado, tratándose además, de una motivación intrínseca que mueve a la acción desde el interés de la persona en un proyecto que le satisface y le ilusiona. La construcción de materiales constituye un elemento motivador que supone una mayor implicación del alumno. Del deseo de construir y jugar 
con los materiales elaborados se desprende una actitud positiva durante todo el proceso, los alumnos se involucran en la tarea desde el principio y muestran interés por las actividades posteriores.

Por otra parte, el hecho de proponerles evaluar su actitud ante el trabajo, en el plano individual y grupal, ha contribuido a fomentar su espíritu crítico por permitirles analizar la diferencia que ha existido en este caso entre lo que debían hacer y lo que realmente han hecho.

A la luz de los datos que aparecen en los cuestionarios de autoevaluación, podemos decir que los objetivos propuestos referidos al trabajo cooperativo se han cumplido en gran medida:

- Clima de cooperación y aceptación mutua

- Responsabilidad ante las tareas individuales y colectivas

- Resolución de conflictos en el seno del grupo

- Potenciación de las relaciones horizontales

Cabe señalar la alta capacidad creativa mostrada en los juguetes, unos diseñados sobre la base de nuestras propuestas, mejorando en gran medida la calidad de las mismas y otros completamente nuevos, fruto de su imaginación y capacidad constructiva. Es de interés resaltar el entusiasmo y la minuciosidad con que se aprestaron al acabado final, haciendo a cada objeto destacar por su originalidad.

Los padres han podido constatar el entusiasmo de sus hijos por el tema al tomar la iniciativa de apartar y guardar envases, al intentar realizar la experiencia con otros niños (amigos, hermanos, primos) y al solicitar, en ocasiones, la colaboración de sus padres en la construcción de sus juguetes.

\section{D.- Evaluación del profesorado sobre la aplicación y viabilidad del programa en el nivel experimentado}

Sobre la viabilidad del programa en el marco escolar los profesores encuestados consideran el programa totalmente viable para llevarlo a la práctica porque enlaza con los objetivos programados en diferentes áreas. Se resalta la utilidad para el desarrollo de los temas relacionados con el medio ambiente, la creatividad y la valoración de los materiales que los alumnos emplearán posteriormente en las clases de Educación Física.

Consideran que resulta idóneo para aplicarlo en el segundo y ter cer ciclo de enseñanza Primaria y el primero de enseñanza Secundaria. Así mismo, reclaman la adaptación del programa para su aplicación en el primer ciclo de Primaria y en el primer ciclo de la ESO, si bien la construcción de ciertos materiales puede realizarse en ciclos inferiores y el material construido puede emplearse en todos los niveles.

Añaden, además, que la secuencia didáctica propuesta permite conseguir plenamente los objetivos señalados. Únicamente destacan que se debería hacer más hincapié en la reutilización de materiales, construyendo fundamentalmente los juguetes que necesitan un menor número de objetos de compra o que, aunque comprados, procedan del reciclado. 
La aplicación de este programa presenta, en la opinión del 85\% de los profesores encuestados, la dificultad de coordinación entre las distintas áreas. Consideran que uno de los factores más interesantes del programa, la interdisciplinariedad, resulta a su vez uno de los aspectos de mayor dificultad.

La recogida y obtención de algunos de los materiales necesarios para la construcción de los juegos es otro de los aspectos destacados como dificultad a la hora de aplicar el programa.

El $85 \%$ de los profesores encuestados consideran adecuado la totalidad del programa. Ahora bien, cabe destacar las aportaciones y modificaciones que presentan algunos profesores por su utilidad para la mejora del programa:

- Realización de una exposición con los materiales construidos y el texto explicativo del juego redactado por los alumnos. Los textos quedarían posteriormente en la biblioteca.

- Utilizar menos materiales comprados.

- Dedicar más tiempo a la puesta en práctica de los materiales utilizados.

En general se enfatiza el planteamiento pedagógico propuesto por el programa como un medio adecuado para el desarrollo integral del niño y el establecimiento de actitudes relacionadas con los objetivos perseguidos en el programa.

\section{E.- Sobre las impresiones del equipo investigador al finalizar el programa}

Hemos podido constatar que el hecho de que el niño sea el artífice de la construcción y elaboración de los materiales que va a emplear en sus juegos, añade multitud de elementos positivos en su actividad escolar, ya que su nivel de implicación y motivación en el proceso lúdico que supone el jugar y elaborar materiales para jugar, ha alcanzado unas cotas elevadas, lo que ha supuesto una grata sorpresa para nosotros.

Podemos concluir, pues, que el programa que hemos elaborado despierta interés y satisfacción en alumnos, padres y profesores y que por ello constituye un instrumento educativo de valía.

Somos conscientes que implica una manera diferente de entender el hecho educativo por el maestro; supone dejar la senda de la disciplinariedad para aventurarse en un planteamiento globalizado del currículo, pero esta experiencia nos confirma la idea de que trabajar sobre proyectos con alta participación en su control por parte del alumnado, con temas cercanos a su mundo de cada día y que exijan el trabajo en equipo, permiten, aparte de poner en práctica varias áreas curriculares, acometer la educación de las actitudes y los valores, aspecto éste que señalan los profesores como deficitario debido a la falta de materiales con que poderlo llevar a cabo.

Manifestar, finalmente, nuestra satisfacción por el resultado final del trabajo. Hacemos nuestra la satisfacción de que hablábamos antes de las personas implicadas en el programa y a ella añadimos la nuestra que tiene su base en el "producto final" de nuestro trabajo, que sería el programa que aquí se ha expuesto y también en el proceso que hemos seguido desde el inicio de nuestra andadura como equipo investigador. Afortunadamente hemos podido constatar que el hecho de ser un grupo interdisciplinar, lo que al principio podría haber supuesto un inconveniente, se reveló como 
altamante positivo ya que nos ha permitido contrastar y compartir puntos de vista diferentes.

Esperamos que esta propuesta sea de utilidad para los maestros, y que redunde, a través suyo, en beneficio para el proceso educativo de los alumnos, que son en definitiva el objeto de nuestro trabajo y nuestro futuro.

ANEXOS

\section{ENCUESTA AL PROFESORADO}

Tipo de Colegio: $\square$ Público $\square$ Privado $\square$ Urbano $\square$ Rural $\square$ CRA

Unidades: $\square$ Infantil $\mathrm{n}^{\underline{0}-} \square$ Primaria $\mathrm{n}^{\underline{0}}-\square$ Secundaria $\mathrm{n}^{\underline{0}}-$ Profesor/a: $\quad \square$ Generalista $\square$ Especialista

Es Tutor/a $\square$ Si $\square$ No Curso:

Área/as que imparte y curso/s:

Años de docencia:

1. Existe alguna demanda o interés, por parte de los alumnos y alumnas, para conseguir un tiempo libre más pleno? ¿Crees que es competencia de la escuela esta labor?

2. Junto al resto de compañeros y compañeras del Claustro, ¿se ha abierto alguna posibilidad para realizar actividades encaminadas hacia una educación del niño en su tiempo libre? ¿Cuáles han sido los resultados derivados de ello?

3. Cómo realizaría la integración del tiempo libre en los contenidos del área? ¿En qué lugar encajaría?

4. Interviene Ud. de alguna manera en las relaciones familiares de sus alumnos comunicándose con los padres y sugiriendo posibilidades de ocupación del tiempo libre del niño y la niña de acuerdo a sus posibilidades?

5. ¿En el PEC de su centro se contempla la creatividad como un objetivo general a desarrollar en todas las áreas?

6. ¿Utiliza alguna técnica en sus clases para fomentar y desarrollar la creatividad? En caso afirmativo, ¿cuáles?

7. Señala los temas transversales que más trabajas en clase y qué cambio de actitudes se observa en los alumnos tras las actividades realizadas

- Educación para el consumo

- Educación para la paz y la convivencia

- Educación ambiental

- Educación vial

- Educación para la igualdad de oportunidades

- Educación moral y cívica

- Educación para la salud y sexual

8. ¿Trabaja en sus clases con algún material de desecho? ¿Con cuál o cuáles? ¿Para qué? 
9. ¿Ha elaborado alguna vez algún material para sus clases de Educación Física? ¿Por qué o para qué?

10. ¿Han participado sus alumnos en la elaboración? ¿De qué forma?

11. ¿Son capaces ellos de diseñar su propio proyecto o necesitan que se les motive?

12. ¿Han participado profesores de otras áreas? ¿De qué forma?

13. ¿Han participado los padres o la APA? ¿De qué forma?

ENCUESTA A PADRES (previa al programa)

Somos un grupo de profesores de la Universidad de La Rioja que Ilevamos a cabo una investigación con la colaboración del centro escolar donde acude su hijo o hija. Intentamos conocer las actitudes de los niños y niñas hacia el Medio Ambiente en el aspecto de la disminución del volumen de residuos y el uso de los materiales de desecho en la construcción de juegos que permitan una ocupación provechosa del tiempo libre y el desarrollo de valores de creatividad, trabajo en equipo, consumo responsable y ocio compartido.

Su opinión previa, junto con la de su hijo o hija, tiene un especial interés para nuestro trabajo. Le agradecemos mucho su colaboración.

1. ¿Tiene costumbre de separar el papel, el vidrio y las pilas del resto de las basuras?

2. Si lleva a cabo la clasificación ¿Cada cuántos días saca estos materiales a los contenedores especiales?

3. ¿Acostumbra a aplastar o romper los envases desechables para que ocupen menos volumen?

4. ¿Da algún uso a los envases desechables? Ponga algún ejemplo.

5. ¿Colabora su hijo o hija en la tarea de separar los materiales?

6. ¿Conocen su hijo o hija los tipos de contenedores? ¿Le encargan a él que lleven los residuos al contenedor correspondientes?

7. ¿Ha visto a su hijo o hija jugar con algún material de desecho?

8. ¿Lo hace actualmente? ¿Qué materiales son los que mas emplea? ¿En qué tipo de juego lo aplica?

9. ¿Le da Ud.expresamente material desechable para que juegue?

10. ¿Cree que lo hacía con mas frecuencia cuando era más pequeño? ¿Qué materiales empleaba? ¿Qué tipo de juegos hacía con ellos?

11. ¿Cree que le daba Ud. más objetos desechables para que jugase?

12. Le parece que fabricando materiales con productos de desecho y jugando su hijo o hija puede manifestar una actitud responsable hacia el problema del volumen de residuos y el despilfarro de energía y materias primas?

13. ¿Le gustaría participar con su hijo o hija en un taller donde se realicen estos materiales? 


\section{ENCUESTA PADRES ( Final del programa )}

Nuestra experiencia en el centro escolar donde asiste su hija o hijo ha concluido y de nuevo pedimos su atención para responder a este cuestionario. Su observación sobre el comportamiento y los intereses de su hija o hijo supone conocer algo de aquello que los niños y niñas hacen y prefieren fuera del contexto escolar y si éste repercute de alguna manera en su vida dentro de la familia y como ciudadano o ciudadana. Esta nueva colaboración supone apoyar la investigación en el difícil campo de la enseñanza y el aprendizaje y, sobre todo, en el de las actitudes. Muchas gracias.

1. ¿Ha contado su hija o hijo algo en casa sobre la experiencia? ¿Qué?

2. ¿Cree que le ha gustado?

3. ¿Ha pedido que le guarden envases?

4. ¿Ha tomado la iniciativa de apartar envases por su propia voluntad?

5. ¿Ha solicitado ayuda para fabricar alguno de los juegos?

6. ¿Ha intentado enseñarle a sus hermanos o amigos?

1a Sesión

\section{PAUTAS PARA LA OBSERVACIÓN}

Lugar: Aula ¡NO LO TIRES, PUEDE SER ÚTIL!

- Los observadores tomarán notas de los argumentos que manejan los alumnos acerca de la elección de unos embalajes u otros. Aportarán las ideas que no surjan.

- Tomarán notas de las posibilidades de reutilización de los envases que proponen los alumnos y añadirá otras nuevas.

\section{PAUTAS PARA LA OBSERVACIÓN}

2a Sesión EXPERIMENTANDO NUEVOS MATERIALES

Clase de Educación Física Trabajo por grupos Observador: . . . . . . . . . . . Fecha................ Curso........ Grupo......... Material.....

1. Comentarios iniciales de los alumnos: lo más curioso (los materiales de desecho utilizados o la forma de construirlos), ¿preguntan cómo se llama? o ¿qué nombre le ponen ellos? ¿por qué? Grado de exploración

2. Forma de agrupamiento (desde el comienzo hasta el final) 
3. Habilidades que se observan. Dominio del material

4. Motivación: tiempo de juego, aplicación a juegos, abandonan, discusiones, juego cooperativo/competitivo

5. Comentarios significativos durante la experimentación

6. Se pregunta a los alumnos al final de la exploración de cada base:

¿Qué habilidades habéis trabajado? ¿Qué otros juguetes podríais hacer a partir de éste?

7. Se pregunta al último grupo que pase por su base: ¿qué material os ha gustado más? ¿por qué?

\section{PAUTAS PARA LA OBSERVACIÓN}

3a Sesión:

\section{CÓMO ES NUESTRO PROYECTO DE TRABAJO}

\section{Observadores:}

Su misión consistirá en orientar a los grupos cuando se crea que la idea que eligen como grupo no se ajusta a sus posibilidades de manejo de materiales o herramientas, o si el juguete no es adecuado para la clase de E.F.

- ¿Cómo es su expresión oral y escrita?

- iTienen en cuenta lo aprendido en sesiones anteriores, en cuanto al medio ambiente ( $1^{\underline{a}}$ sesión) y la forma de ensamblar, cortar, unir, reforzar, etc. de la $2^{\underline{a}}$ sesión?

- ¿Participan en los grupos todos los componentes?

- ¿Hay discusiones? ¿Cuáles son el tono y los motivos?

- ¿Hay ambiente de trabajo? 


\section{PAUTAS PARA EL PROYECTO DE JUGUETE}

Describid el objeto: ¿Cómo es? ¿Qué tamaño tiene? ¿A qué se puede jugar con él? Haced los dibujos necesarios: visto desde arriba, desde abajo, de perfil, en perspectiva....

Haced una lista de los objetos que vais a reutilizar

Haced una lista de los materiales que os harán falta

Haced una lista de las herramientas que utilizaréis

Escribid las tareas que deberéis realizar y quién se encargará de ellas

\section{PAUTAS PARA LA OBSERVACIÓN}

4 Sesión:

REALIZANDO NUESTROS PROPIOS MATERIALES

Lugar: Taller de Plástica.

Duración: 2 horas

Pautas de observación:

- Comentarios iniciales de los alumnos.

- Nivel de motivación de los alumnos

- Comentarios significativos durante la experimentación.

- Número de modificaciones con respecto a lo proyectado:

+ en las tareas

+ en la adjudicación de tareas

+ en las medidas

+ en los materiales

- Necesidad de dirección en la realización de materiales.

- Observación del nivel de habilidades y dificultades que presentan los alumnos al realizar las tareas.

- Dinámica de grupos: problemas en la coordinación del equipo, discusiones, abandonos

- Aparición de problemas en la construcción

- Creatividad

- los materiales que diseñan

- su construcción

\section{AUTOEVALUACIÓN PARA ALUMNOS}

\section{VALORAD VUESTRO JUGUETE}

1. Comparad el juguete que habéis construido con el que diseñásteis. Para ello utilizad el proyecto que elaborásteis. ¿Cuáles son las diferencias?

2. Valorar los acabados y los aspectos estéticos: ¿Ha quedado bonito? ¿Está bien rematado? 
3. Te resultó divertido jugar con los materiales que os presentamos?

4. Estaban bien construidos? ¿Se estropearon con el uso?

5. Valorad la utilidad del juguete: Os ha sido útil para mejorar en

6. Cómo se podría mejorar el juguete?

\section{AUTOEVALUACIÓN PARA ALUMNOS}

\section{VALORAD VUESTRO TRABAJO INDIVIDUAL}

- ¿He sido responsable en mis tareas?

- ¿He aportado ideas?

- ¿He participado en la toma de decisiones?

- ¿He respetado las opiniones de los demás?

\section{VALORAD VUESTRO TRABAJO EN GRUPO}

- ¿Habéis tenido problemas al decidir el juguete que queríais construir?

- ¿Qué sistema habéis empleado para tomar la decisión si teníais más de una idea?

- ¿Habéis organizado bien el trabajo?

- ¿Habéis trabajado a gusto? ¿Por qué?

- Si tuviérais que hacer otro trabajo en grupo ¿Qué cambiaríais?

\section{Referencias Bibliográficas}

ALVIRA MARTíN, F. (1991). Metodología de la evaluación de programas. Madrid: CIS ARCA,J., DELPUY, E., LLANAS, T. Y ROY, Y. (1997). Viaje al interior del cuerpo humano: una colonia para el tiempo libre. Madrid: CCS

BARRENECHEA, P.; LARRUGA, S. y VAREA, M. (1997). Basureros y vertederos de Aragón. Zaragoza: Mira editores

BRAVO BERROCAL, R. y ROMERO RAMOS, O (1998). Actividades educativo-complementarias en la naturaleza. Granada: Aljibe

CAMPBELL, D.T (1982). Grados de libertad y el estudio de casos, en COOK, T.D. y REICHARDT, Ch. S. (1986) Métodos cualitativos y cuantitativos en investigación evaluativa. Madrid: Morata

DE LA ORDEN, A. (1985) Investigación evaluativa, en DE LA ORDEN, A. (dir) Investigación Educativa. Diccionario de Cas. Educación (pp133-137). Madrid: Anaya

FILSTEAD, W.J. (1982). Métodos cualitativos. Una experiencia necesaria en la investigación evaluativa, en COOK, T.D. y REICHARDT, Ch. S. (1986) Métodos cualitativos y cuantitativos en investigación evaluativa. Madrid: Morata 
GONZÁLEZ FARACO, J. C. (1997). Verde que te quiero verde...Un análisis crítico de los modelos hegemónicos en la Educación Ambiental, en Bordón 49 (4), (pp. 435-444).

GONZÁLEZ LUCINI, F. (1994). Educación, ética y transversalidad, en Cuadernos de Pedagogía no 227 (pp. 10-13).

GUTIÉRREZ PÉREZ, J. (1995) La Educación Ambiental. Fundamentos teóricos, propuestas de transversalidad y orientaciones extracurriculares. Madrid: La Muralla.

MOPMA (1995). Medio ambiente en España. Madrid.

PONCE DE LEÓN ELIZONDO, A. (1998). Tiempo libre y rendimiento académico. Logroño: Universidad de La Rioja

PONCE DE LEÓN ELIZONDO, A. (1997). "Reflexiones y conclusiones sobre el tiempo libre y los valores", en Revista Bordón no 49. (pp. 87-94)

PONCE DE LEÓN ELIZONDO, A. y GARGALLO IBORT, E. (Coor) (1999a). Reciclo, construyo, juego y me divierto. Una propuesta interdisciplinar para la Educación del Ocio, el Consumo, el Medio Ambiente y la Educación Física. Madrid: CCS

PONCE DE LEÓN ELIZONDO, A.; GARGALLO IBORT, E.; LEMUS VARELA, M.C.; LOZA OLAVE, E.; FERNÁNDEZ ARMESTO, M.L.; TREVIÑO FERNÁNDEZ, M. P. Y PASCUAL SUFRATE, M.T. (1999 b) Experiencia interdisciplinar: innovación en la escuela y en la formación inicial del profesorado, en Revista Interuniversitaria de Formación del Profesorado (En prensa)

RUTMAN, L. (1984) Introduction, en RUTMAN, L. (ed) Evaluation research methods: a base quide. London:Sage

TONUCCl, F. (1988). A los tres años se investiga. Barcelona: Hogar del libro S.A.

UNESCO Programa Internacional de Educación Ambiental (1994). Actividades de Educación Ambiental para la enseñanza Primaria. Ed. Los libros de la Catarata. Gobierno de La Rioja.

VELÁZQUEZ CALLADO, C. (1996). Actividades prácticas en Educación Física. Cómo utilizar materiales de desecho. Madrid: Escuela Española.

V.V.A.A. (1994). La respuesta curricular, en Cuadernos de Pedagogía no 227 (pp. 1418).

YUS, R. (1996). Temas transversales. Hacia una nueva escuela. Barcelona: Grao.

YUS, R. (1996). Hacia una educación global desde la transversalidad. Madrid: Anaya Alauda. 